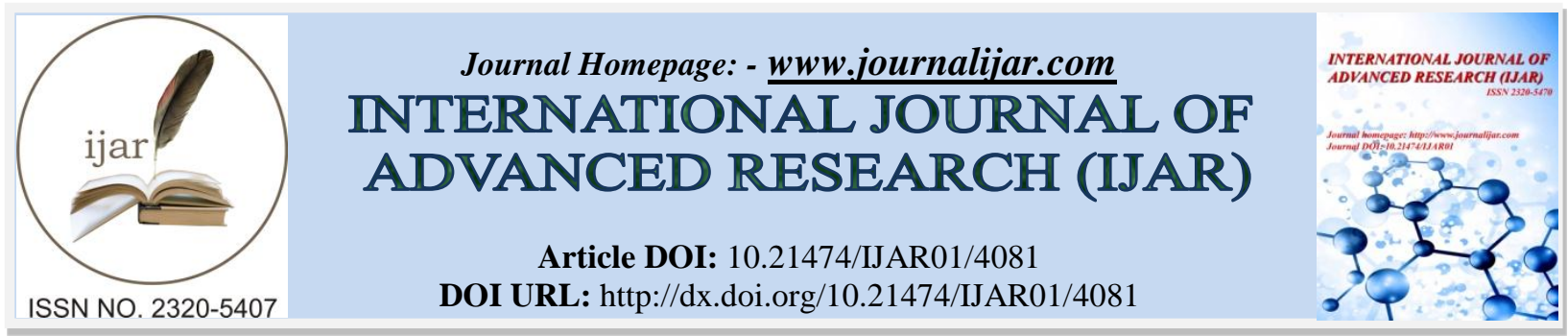

RESEARCH ARTICLE

\title{
MOTHERS PERCEPTION OF BLOOD TRANSFUSION IN CHILDREN UNDER 5 YEARS IN KISORO HOSPITAL, UGANDA
}

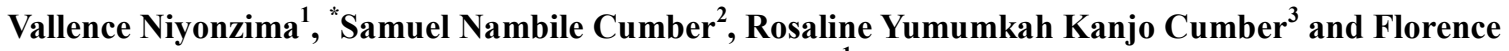 Beinempaka ${ }^{1}$}

1. Department Mbarara University of Science and Technology (MUST), Uganda.

2. Discipline of Public Health Medicine, Department of Nursing \& Public Health, College of Health Sciences, University of Kwazulu-Natal, Durban, South Africa.

3. Department of Political Science, University of KwaZulu-Natal, Durban, South Africa.

\section{Manuscript Info}

\section{Manuscript History}

Received: 03 March 2017

Final Accepted: 01 April 2017

Published: May 2017

Key words:-

Blood transfusion, perceptions, qualitative descriptive design

\section{Abstract}

Introduction: Blood transfusion is the main cornerstones of modern medical practice which started way back in 1818 by James Brundell (Virge et al, 2000) and is the main treatment for anaemia. Although it is an important clinical intervention in paediatrics, surgery, maternal health and general medicine, it has been received with mixed feelings from the mothers sometimes leading to runaways or direct rejection.

Objective: This study sought to explore mothers' perceptions of blood transfusion in children under five years.

Methodology: A qualitative descriptive design was employed. The study involved seven mothers selected by purposive sampling, who were caring for medically stable children who had received a blood transfusion. Data was coded, transcribed and analysed using Colaizzi's approach.

Results: Four themes emerged from nine categories desccribing mothers' perceptions of blood transfusion: Trust, fear of the unknown, blood safety and being optimistic. These qualitative findings reveal mixed feelings about blood transfusion.

Conclusion: In conclusion the themes that emerged were mostly expressions of perceptions of blood transfusion. These qualitative findings suggest that public acceptance of blood transfusion as a medical intervention is based on their perceptions of the risks involved and the perceived benefits of the intervention.

Recommendations: It is clear that knowledge of the facts about blood transfusion by health workers is paramount; therefore this knowledge needs to be communicated to the public so that they are able to make informed choices about their treatment.

There should be psychological preparation of the patients and their caretakers prior to blood transfusion in an attempt to change the negative perceptions and alleviate fears.

Copy Right, IJAR, 2017,. All rights reserved.
Corresponding Author:- Dr. Samuel Nambile Cumber.

Address:- Discipline of Public Health Medicine, Department of Nursing \& Public Health, College of Health Sciences, University of KwaZulu-Natal, Durban, South Africa. 


\section{Introduction:-}

Blood transfusion is the main cornerstone of modern medical practice which started way back in 1818 by James Brundell (Virge et al, 2000). It is an important clinical intervention in the health care delivery especially in surgery, general medicine, maternal health and child health. In child health malaria has been the most condition that necessitates blood transfusion (WHO, 2005).

In a comparative study by de Graaf et al (2009), he reported that $10 \%$ of all hospital stays in the United States in 2008 received blood transfusion while in the Netherlands in 2006, 556,509 units of blood were administered and in Uganda 135,000 units were administered. However as observed by de Graaf et al (2009), the various outcomes of transfusions performed here in Uganda are hard to come by due to poor documentation.

Most of the paediatric transfusions in Uganda have been as a result of anaemia due to malaria infections but this has not been the case in Kisoro due to cold conditions which did not favour mosquito survival in the area. Of recent, however, the reverse has been experienced due to climatic changes and the number of malaria cases among the children is on the rise.

The few transfusions that have been done in Kisoro have been received with mixed feelings from the mothers and caretakers, some times resulting in runaways or direct rejection by the mothers and/or caretakers. Nevertheless, blood transfusion remains the main treatment option in children with severe anaemia ( WHO, 2005).

Some mothers have been heard advising their friends not to accept a blood transfusion once it has been prescribed for the child. As a result some mothers excuse themselves to do other things, carrying the sick children at their backs when it is time to administer the prescribed blood transfusion.

A few mothers accept the prescribed blood after a series of counseling sessions by the nurses and doctors. Others run away with their children or reject the blood directly. For those who accept, in case more than one unit is prescribed, the second one is most of the time rejected. Therefore the purpose of this study is to explore mothers' perception of blood transfusion in children under five years.

The objective of this study was to explore mothers' perception of blood transfusion in children less than 5 years admitted in Kisoro hospital.

The study findings will help health workers devise ways of providing information to mothers regarding blood transfusion in an attempt to change their negative perceptions towards blood transfusion.

This study will generate information which can be used in nursing education to increase scope of knowledge on blood transfusion and perceptions of mothers towards blood transfusion.

The study will provide an opportunity or reference materials for further research to be carried out about blood transfusion in children less than five years.

\section{Methodology:-}

\section{Introduction:-}

This chapter explains the various methods and procedures that were used and followed while conducting the study. These include the research design, study setting, study population, sample determination, sampling procedure, inclusion criteria, exclusion criteria, definition of variables, research instruments, data collection procedures, data management, data analysis, ethical consideration, limitations of the study and dissemination of results.

\section{Study setting:-}

The study was conducted in Kisoro hospital. Kisoro hospital is located in Kisoro district in south western Uganda and borders Rwanda and DRC and therefore receives patients from the local population and the neighboring countries. The district is according to the human poverty index (HPI), the fifth poorest in Uganda. This district also has a high illiteracy rate (50.9\%) compared with the national level (38.4\%), (UNDP 1999). It is heavily affected by malaria especially during the rainy season (march-June, September- November). Kisoro hospital is a government 
hospital, hence receives patients of a low economic status. The study area was chosen because the researcher is well versed with the language.

\section{Study design:-}

The study employed a qualitative descriptive design. This research design was chosen because it explained and described phenomenon; and allowed for in-depth exploration of issues of interest (Burns \& Grove, 2003) which in this case was "Mothers' perception of blood transfusion of children under 5years".

\section{Study population:-}

The study population comprised of mothers caring for an under 5year old child receiving blood transfusion.

\section{Sample size:-}

The sample size was determined by the saturation point. Saturation of the data occurred when additional sampling provided no new information, (Burns \& Grooves, 2003)

Saturation method was used because it helped the researcher to exhaust all the relevant data about the phenomenon.

\section{Sampling technique:-}

Purposive sampling technique was employed. The purposive technique was chosen because it provided information rich case (Norwood, 2002) and the nursing situation that the study was investigating required the same. Identification of these mothers was done by use of the patients' charts, nursing officers and other staff members in Kisoro hospital.

\section{Inclusion Criteria:-}

Selection of participants was limited to the mothers who:-

- Were caring for a child on blood transfusion and were still on the ward.

- Consented to participate.

- Were able and willing to describe their perceptions about blood transfusion and express their inner feelings

- Were able to express themselves in the local language (Rufumbira) and/or English

\section{Exclusion criteria:-}

The study excluded mothers;

- Whom the researcher knew personally

- Who had very sick children

\section{Data collection Tool:-}

- Data was collected using an in-depth tape recorded interview

- An in-depth interview guide was also used because it allowed for a detailed exploration of information from the respondents and freedom of self-expression and had high chances of generating valid information.

\section{Data collection procedures (technique):-}

The researcher first reviewed documents relating to perceptions about blood transfusion. Then later, an in-depth interview guide with an open ended question was used to collect data from the respondents. This was tape recorded. After collecting data, the researcher organized the collected data and processed it for final analysis.

\section{Data Management and analysis:-}

1. Data analysis was done and participants were assigned codes P1-P7 to ensure confidentiality. Data analysis was done using Colaizzi's (1978) approach. The approach helped to generate data that require formulated descriptions that was proved by the participants of the study.

2. This followed the seven steps below;-

3. The written descriptions of the perceptions of the respondents were carefully read and re-read by the researcher to ensure familiarity. This process was facilitated by the researcher immersing himself in the process, while reading and re-reading the transcribed information and also listening to the recorded information.

4. Participants' attention was required to facilitate further understanding of the mothers' perceptions as the researcher immersed himself in the descriptions, phrases or sentences that pertain to the phenomenon under 
study were extracted. This step is referred to as "extracting significant statements", also referred as thematic statement.

5. The meaning of each "significant statement" was clarified; meanings were formulated, while clarifying. Caution was taken at this stage not to change the meaning from the original written descriptions.

A. Organization of the formulated meanings from all the descriptions (protocols) into clusters of themes, sub themes was involved in this fourth step:

B. The researcher determined information in the cluster themes, newly identified themes were referred back to the original description for validation. Here the researcher determined if there is any information in the original written descriptions that was not accounted for in the cluster of themes. The researcher also determined whether the cluster of themes propose anything that was not implied for in the original protocols. It is at this stage that all clusters of themes were validated by the researcher for correctness.

6. The discrepancies among the various clusters were taken into account, while not ignoring the themes which do not fit anywhere with clusters.

7. An exhaustive description of the phenomenon that was investigated was carried out. From the identified thematic statements, the researcher formulated a description of the experience that best exemplified the essence of the perceptions as they manifested in the participants' story. This was developed from the result of the data analysis.

8. The researcher made an effort to describe the investigated phenomenon in unambiguous and explicit terms as possible to enhance the understanding of the phenomenon.

9. To achieve final validation each respondent was given exhaustive description of the phenomenon and the participant was asked whether or not the description arrived at fully described her perceptions. The respondents were asked if any aspects of their perceptions were omitted. Any relevant data obtained from the subjects during the validation was incorporated into the final product of research.

Analysis began as soon as the first data was collected and went on concurrently with data collection.

\section{Ethical Consideration:-}

The proposal was submitted to the faculty of medicine research ethics committee (FREC) for ethical approval. An introductory letter was obtained from the department of nursing and submitted to the institution where the study was conducted seeking permission from the hospital director to carry out research. Participants were told that participation in the study was purely voluntary and that they were free to withdraw at any time they wished and this would not affect their services. They were told that the information gathered would only be used for the purpose of study and it would be kept with confidentiality.

\section{Study Limitation:-}

This being a qualitative study, few participants were interviewed and therefore the findings cannot generalized but only transferable.

Since human beings were the instruments for data collection, findings can be affected by individual competency. Limited literature on the topic especially on local data made it difficulty.

\section{Rigors of research:-}

Rigors relate to establishment of trustworthiness of the data and study findings. According to Polit \& Hungler (1998), the factors to be considered in assessing the trust worthiness of research are.

\section{Credibility:-}

Credibility aimed at increasing the truth and value relating to the findings of the study and was achieved through Member checks and prolonged engagement during the interview (Finley, 2003).

Questions were adequately explained to the respondents during the interview for purpose of understanding to avoid vague answers. Credibility was also enhanced by ensuring strict selective criteria stipulated above.

In this study, the researcher achieved credibility using one-to-one in-depth interview which allowed participants reveal their perceptions about blood transfusion. Participants were allowed to take their time narrating their experiences 


\section{Dependability:-}

This was ensured by careful listening to the recorded responses and data analysis was audited which was preceded by audit trails of the verbatim transcription of the words and phrases as they were used to describe mothers' perceptions of blood transfusion in children under 5 years.

\section{Conformability:-}

Ways of how interpretations were arrived at were shown. If credibility, dependability were ensured then conformability was found suiting.

\section{Dissemination of results:-}

Final copies of the study were produced after approval. One report was submitted to Mbarara University of Science for partial fulfillment of a Bachelor's degree in nursing science, a copy to MUST Library, Department of Nursing, Study area and another copy was retained by the researcher.

\section{Results:-}

\section{Introduction:-}

This chapter presents findings on mothers' perception of blood transfusion in children under 5years in Kisoro hospital. Four main themes that emerged from nine categories were: trust, fear of the unknown, blood safety and being optimistic. Description of the phenomenon was developed, incorporating the thematic categories into substantive meaning of the mothers' perception regarding blood transfusion.

\section{Demographic characteristics of the participants:-}

The study involved seven participants whose age range was 21-37. Five of them were Catholics and 2 were Protestants. All the participants were Bafumbira. Four of the participants had acquired primary education; one had gone as far as secondary level while two had never gone to school.

\section{Theme one: Trust:-}

This theme emerged from three categories: complete cure, saves life and full recovery.

\section{Complete cure:-}

This is depicted from responses of participants P4, P1, P3 and P5.

... I know if someone is added blood there is a way his/her body has failed, therefore the added blood will help cure them...

...The added blood helped to cure my child...

... I believe what health workers do is to treat and cure my child...

... My son became somehow okay in two hours after giving him blood...

\section{Saves life:-}

Participants described blood transfusion as an intervention that saves children's lives as stated by participants P2, P4 and $\mathrm{P} 7$

...I know blood saves life...

... It was the blood that saved my child's life...

...I know blood has saved lives of many...

One participant (P7) expressed concern that the prescribed blood was being delayed yet it was intended to save her child's life

... These nurses are delaying to bring blood.... my child is going to die instead of being saved. What do I do? ...

\section{Full recovery:-}

The participants (P2, P4, P6, P3 and P7) also described blood transfusion as an intervention that helps patients to improve and fully recover.

... I have seen children becoming better on blood...

... Others die if not given blood...

... Adding blood improves a sick child's life...

... For someone to be added blood it means he/she doesn't have enough blood...

... The added blood helps to improve their condition...

One participant (P3) affirms that blood transfusion can assist children recover from recurrent illnesses. 
... When a child is given blood, he or she does not fall sick now and then...

Theme Two: Fear of the unknown:-

This theme originated from three main categories: fear, hasten death and worsen the condition.

Fear:-

Participants expressed a lot fear regarding the administration of blood transfusion and were concerned that the blood might be too much for the child and cause other problems (P3 and P6)

... I thought that bag of blood was too much for my baby...

... This is a third unit ... really it's beyond...

One participant (P2) was concerned why the child was being transfused when it had not bled at all.

... I have not seen my child bleeding... I think this is too much for him...

\section{Hasten death:-}

Participants (P4, P2, P5 and P1) expressed fear that blood transfusion may fasten death.

... I fear that when a patient is put on blood he/she is going to die...

... My first born died immediately she was added blood, so I still fear the same can happen...

... When I saw blood hanged on my baby, eeh!! I thought time has reached for her to die...

... I realized he was going to die so I prayed...

\section{Worsen the condition:-}

Two participants (P5 and P7) attributed the deterioration of patients' condition to blood transfusion

... Others are put on blood (keeps quiet for a moment) they do not improve so they die...

... My son's situation even became worse when he was added blood...

... Others' stomachs' enlarge when added blood and they cannot even breathe thereafter...

Theme Three: Blood safety:-

This theme emerged from one category; fear of contracting diseases.

\section{Fear of diseases:-}

Participants showed a lot of worries concerning the safety of the blood that is used for transfusion. Most of them (P1, P3, P4, P7 and P6) were concerned about their children contracting HIV/AIDS from the blood.

... I had a lot of worries because... they could have diseases more so "sirimu" which is common these days...

... During this era of AIDS, it becomes tricky to accept blood from every one. You may get infected...

... How can you know that this blood does not contain germs? I fear my child may get infected...

...I fear that my innocent child will get diseases from this blood...

...My child can easily get AIDS from this blood...

\section{Theme Four: Being optimistic:-}

This theme emerged from two categories; being hopeful and having too much confidence in health workers.

\section{Being hopeful:-}

Two participants (P2 and P5) expressed hope that their children were going to be better once given blood.

... I feel strong and believe that God is on our side...

... When my child is very sick and is put on blood, I get hope that he will improve...

Too much confidence in health workers:-

Participants (P1, P4, P6, P3 and P5) demonstrated too much confidence in health workers; they think every intervention a health worker does is good for the child.

...I know the health workers always do what is good for the child...if they decide to add blood, it is okay...

...you take your child to the hospital to be helped. If the doctor decides the child blood, then you accept...

... If my child did not really need it, they wouldn't have said she did...

... I took their word that blood transfusion was necessary...

... Two doctors conferenced and made the decision... I think my child really needed the blood... 


\section{Discussion:- \\ Introduction:-}

This chapter presents discussion of findings, the description of phenomenon and focused literature review on concepts related to the thematic structures of mothers' perceptions of blood transfusion in children less than 5 years. There are four main themes that emerged from nine thematic categories that describe the phenomenon of mothers' perception of blood transfusion in children under 5 years.

\section{Trust:-}

Trust refers to a firm belief in someone or something. This was expressed by participants in their responses such as $I$ believe what health workers do is to treat and cure my child, it was the added blood that saved my child's life and I have seen others improving on blood. This finding is in line with that reported by Budack et al (2006) whereby 18\% of the respondents acknowledged that blood saves life and therefore would accept a blood transfusion whatsoever. Andrews (2010) noted that certain types of illnesses and medical conditions cause temporary but profound anemia and therefore patients with these conditions require blood transfusion to correct the anemia. This reduces mortality due to anemia. He further revealed that transfusions in patients with bone marrow failure help to sustain life. In such conditions blood transfusion is very vital as far as saving life is concerned and thus is trusted by the patients or caretakers.

\section{Fear of the unknown:-}

Participants demonstrated a feeling of anxiety concerning the outcomes of the blood transfusion. Some thought it would worsen the condition or quicken death and others generally hard a lot of fear owing to the fact that blood transfusion is a procedure that is rarely performed in this setting.

This finding is in line with that of de Graaf et al (2011) who, in his observational study in Mulago, noticed that the general condition of some patients deteriorates and others died after a blood transfusion.

Adams et al (2011) noted that the fear regarding the outcome of blood transfusion has been accelerated by the advancement in technology where information regarding adverse transfusion reactions can be easily accessed by the participants. Therefore, they may end up refusing blood transfusion even when they are aware that it is a lifesaving intervention.

\section{Blood safety:-}

It was found out that most of the participants had doubts about the safety of the blood used for transfusion. They had fear of their children contracting diseases from the blood especially HIV/AIDS since it is through blood that that these infections are spread. This finding is in agreement with the results of a related study conducted by Luby et al (2001) who found out that $20 \%$ of the respondents would refuse blood transfusion even if they were in need because of the risk of the risk of acquiring an infectious disease. The same study revealed that $55.1 \%$ of the participants revealed that blood in blood banks was unsafe. Another study conducted in Namibia by Budack et al (2006) revealed that participants revealed fear of contracting HIV/AIDS, hepatitis, malaria, STDS and Diabetes. Luby et al (2001) also noted that there is a risk of acquiring infection from the blood since it may contain germs and other disease causing organisms. Similarly Finucane (1998) also found out that a substantial proportion of the US population did not consider the US blood supply safe. Therefore doubts about the safety of blood have increased during this era of HIV/AIDS and this has reduced the acceptance of blood transfusion.

\section{Being optimistic:-}

Participants expressed a lot of hope and confidence in the health workers. This was expressed in their responses such as When my child is very sick and is put on blood, I get hope that he will improve, I feel strong and believe that God is on our side, I know the health workers always do what is good for the child, if they decide to add blood, it is okay, you take your child to the hospital to be helped. If the doctor decides the child blood, then you accept, if my child did not really need it, they wouldn't have said she did. This finding is contrary to what was reported by Adams et al (2011) who noted that blood transfusion is always associated with fear due to the uncertain outcome. This is attributed to the fact that caretakers have seen patients on blood transfusion improve and recover and therefore patients and caretakers accept a blood transfusion whatsoever once it has been prescribed. 


\section{Conclusions}

In conclusion, the four themes; trust, fear of the unknown, blood safety and being optimistic that emerged from nine categories were mostly expressions of perceptions of blood transfusion. These qualitative findings suggest that public acceptance of blood transfusion as a medical intervention is based on their perceptions of the risks involved and the perceived benefits of the intervention. These findings were from seven participants who participated voluntarily in the study.

\section{Recommendations:-}

The results from the interviews held with mothers regarding their perceptions about blood transfusion in children less than 5 years revealed mixed feelings. Recommendations to address this are the following:

It is clear that knowledge of the facts about blood transfusion by health workers is paramount; therefore this knowledge needs to be communicated to the public so that they are able to make informed choices about their treatment.

The health workers should examine current practices and improve procedures so that patients receiving blood transfusions get more support, as well as more information on the need for transfusions and on alternatives that might be available.

There should be psychological preparation of the patients and their caretakers prior to blood transfusion in an attempt to change the negative perceptions and alleviate fears.

\section{Nursing implications:-}

The results from this study contribute to the understanding of mothers' perception of blood transfusion. The study lends evidence to our practice by providing information about how it is important for health care providers to provide information regarding different procedures to enhance understanding by patients and caretakers.

In practice, nurses form a vital interface between the patients and the health system. They hold a body of information which if well dispensed could have a great implication to change the perceptions about blood transfusion and improve its acceptance by the public.

It is important to advocate for hospital policy that includes providing support for patients and caregivers. Nursing guidelines should be developed for quality care of these patients.

Therefore there is a need to educate nurses on the blood transfusion procedure and specific interventions for the care of patients receiving blood transfusion.

\section{Areas for further research:-}

Similar studies should be done in other parts of the country and the results compared which will help in strengthening health education about blood transfusion and prevention of malaria

Since this is a qualitative research, quantitative research should be carried out on assessment of the knowledge of mothers and other caretakers on blood transfusion.

Also a research about psychological preparation of patients and caretakers by health workers before blood transfusion using qualitative or quantitative designs should be done.

Evaluating the whole process of blood transfusion from the time blood is prescribed, how blood is transported, monitoring and documentation done. 


\section{Reference:-}

1. Adams K.W. and Tolich D. (2011): improving the patient's experience of blood transfusions. American journal of nursing 2011;111:24-30

2. Andrews M.M. and Boyle J.S. (2003): Transcultural concepts in nursing $4^{\text {th }}$ edition Philadelphia, U.S.A pages 470, 481

3. Bhopal R.S., McEwan R.T., Madhok R. and McCallum A. (1993): Perceptions about blood donation, transfusion and the risk of HIV infection: Implications for the blood transfusion services: AIDS care 1992; 4(1): 43-52

4. Budack R., Sylivia T., Liesel S., Kamau J. and Chitsva J. (2006): Study on the knowledge, beliefs, perceptions, attitudes and practices on voluntary non- remunerated blood donation and transfusion in Namibia, Ministry of Health and social Services, Namibia.

5. Burns N. and Groove S.K. (2003): Understanding nursing research $3^{\text {rd }}$ edition. W.B Saunders, Philadelphia P.A.

6. De Graaf J, D., Kajja I., Bimenya GS, Postma M,J., Sibinga C,T (2011): Bedside practice of blood transfusion in a large teaching hospital in Uganda: An observational study. Asian J Transfus Sci 2009;3:60-5

7. Ferguson, E., Farrell, K., Lowe, K. C. and James, V. (2001): Perception of risk of blood transfusion: knowledge, group membership and perceived control. Transfusion Medicine, 11: 129-135.

8. Finucane B.P: Public perception of the risk of blood transfusion 2000; 40:1017-1022

9. Fitzgerald M. (2000) Blood transfusion from the recipient's perspective. J Clin Nurs 1999;8(5):593-600.

10. Hodgkinson B, (1999). Towards safer blood transfusion practice. J Qual Clin Pract 1999;19 (1):63-7

11. Hollinger FB, Kleinman S: Transfusion transmission of West Nile Virus: a merging of historical and contemporary perspectives. Transfusion 2003, 43(8):992-997

12. Hossenlopp, C. (2001): The risk debate in blood transfusion: how perceptions, beliefs and behaviors can be shaped by an efficient communication. Transfusion Medicine, 11: 124-12

13. Kleinman S, Chan P, Robillard P: Risks associated with transfusion of cellular blood. Transfusion Medicine Reviews 2003, 17(2):120-162

14. Lee D.H, (2004). Differences in the perception of blood transfusion risk between laypeople and physicians. Transfusion 2003;43(6):772-8.

15. Luby S.P., Niaz Q., Siddiqui S., Mujeeb S.A. and Fisher- Hock S. (2001): Patients perceptions of blood transfusion risks in Karachi, Pakistan. Int J Infect Dis. 2001;5(1):24-6

16. Politis C., Richardson S.C. Perception of the risk of HIV infection through giving blood in Greece Vox Sang 1997;72:251- 252.

17. Potter P. and Perry A.G. (2005): Fundamentals of nursing, Mosby Elsevier, St. Louis

18. Rawn J. (2008): The silent risks of blood transfusion. Curr Opin Anaesthesiol 2008; 21: 664-8

19. Trop J.P. Hospital users' knowledge about blood transfusion and awareness and attitudes towards HIV/AIDS infection in a region of Ghana. J Trop Paediatr 1992; 38(2) 94-95

20. Watson-William E, J., Kataaha P., Ssenyonga P.(1991): Development of Uganda blood transfusion services, Kampala, Uganda.

21. Whittaker S. (2003): Qualitative research: what is it and how can it be applied to transfusion medicine research? Vox Sang 2002;83 Suppl 1:251-60.

22. WHO (2005): World malaria report. Geneva 